\title{
Supplementary Material to paper 'Systematic comparison of five machine-learning methods in classification and interpolation of soil particle size fractions using different transformed data' \\ Mo Zhang, Wenjiao Shi
}

5

This supplementary material consists of equations of methods, tables and prediction maps in the paper and in the following 5 sections. Section $\mathbf{S 1}$ is the equations description of machine-learning models. Section S2 shows parameter adjustment of machine-learning models. Section S3 includes the uncertainty assessment of soil PSFs interpolation. Section S4 includes the prediction maps of silt and clay fractions. Section S5 demonstrates the indirect classification maps using ALR and CLR transformation methods.

\section{Supplementary Material}

\section{Section S1 The equations description of machine-learning models}

For K-nearest neighbor (KNN), for a train set of observed data $L=\left\{\left(y_{i}, x_{i}\right), i=1, \ldots, n_{L}\right\}$, class $y_{i} \in$ $\{1, \ldots, c\}$, and the predictor values $x_{i}^{\prime}=\left(x_{i 1}, \ldots, x_{i p}\right)$. For a new observation $(y, x)$, the nearest neighbor $\left(y_{(1)}, x_{(1)}\right)$ is based on the distance function which is as follows:

$d\left(x, x_{(1)}\right)=\min _{i}\left(d\left(x, x_{i}\right)\right)$

and $\hat{y}=y_{(1)}$ refers to the nearest neighbor, which is the prediction for $y$. Value $x_{(j)}$ and $y_{(j)}$ are the $j$ th nearest neighbor of $x$ and class of training set, respectively.

For multilayer perceptron neural network (MLP), each neuron $j$ sums input environmental covariate in our study $x_{i}$ after multiplying them by the connection weights $w_{j i}$ respectively, and calculates its output $y_{j}$ (soil PSFs components or texture class) as a function of the sum:

$y_{j}=f\left(\sum w_{j i} x_{i}\right)$,

25 where $f$ is the activation function, which can be a linear or logistic function. The sum of squared differences between the predicted values and observed values of the output results of neurons $E$ is defined as follows:

$E=\frac{1}{2} \sum_{j}\left(y_{p j}-y_{o j}\right)^{2}$

where $y_{p j}$ and $y_{o j}$ is the predicted and observed value of output neuron $j$, respectively. Each $w_{j i}$ is adjusted to reduce $E$ and the adjustment of $w_{j i}$ depends on the training algorithm.

For random forest (RF), the equations for Gini index and minimizing the sum of the squares of the mean deviations $(\mathrm{M})$ are as follows:

Gini $=1-\sum_{k=1}^{K} p_{k}^{2}$,

$\operatorname{Gini}(D, A)=\frac{\left|D_{1}\right|}{|D|} \operatorname{Gini}\left(D_{1}\right)+\frac{\left|D_{2}\right|}{|D|} \operatorname{Gini}\left(D_{2}\right)$,

$\mathrm{M}=\min _{A}\left[\min _{c_{1}} \sum_{x_{i} \in D_{1}(A)}\left(y_{i}-c_{1}\right)^{2}+\min _{c_{2}} \sum_{x_{i} \in D_{2}(A)}\left(y_{i}-c_{2}\right)^{2}\right]$,

where $p_{k}$ refers to the proportion of $k$ th class in the data set on the current node, for feature $A=a$, data set $D$ is divided into two parts $\left(D_{1}\right.$ and $\left.D_{2}\right), D_{1}$ describes the data set which meets the condition 
$A=a$ and $D_{2}$ is the opposite of $D_{1} ; \operatorname{Gini}(D, A)$ represents the uncertainty of set $D$ after binary split; $y_{i}$ is the predicted value of input value $x_{i} ; c_{1}$ and $c_{2}$ is the mean of data set $D_{1}$ and $D_{2}$, respectively.

In support vector machine (SVM), for a data set $\left\{x_{i}, y_{i}\right\}, i=1, \ldots, k, x \in R$ and $x$ refers to an ndimensional vector, $y \in\{-1,+1\}$ is the class corresponding to $x$, the equation for calculating a 5 hyperplane of SVM is defined as follows:

$\min _{w, b, \xi} \frac{1}{2} w^{T} \times w+C \sum_{i=1}^{k} \xi_{i}$,

s.t. $y_{i}\left(w^{T} \times \phi\left(x_{i}\right)+b\right) \geq 1-\xi_{i}, \xi_{i} \geq 0, i=1, \ldots, k$,

where $\phi\left(x_{i}\right)$ refers to the mapping from the input space to the feature space, $C>0$ is penalty factor (cost), $w, b$, and $\xi$ are the parameters need to be optimized during the process of model training, which can be determined by the Lagrange multipliers:

$f(x)=\operatorname{sgn}\left(y_{i} a_{i} k\left(x_{i}, x\right)+b^{*}\right)$,

where $a_{i}$ refers to the support vector, $k\left(x_{i}, x\right)$ refers to the kernel function, and $b^{*}$ is the bias.

For extreme gradient boosting (XGB), the general prediction function at step $\mathrm{t}$ is defined as follows:

$f_{i}^{(t)}=\sum_{k=1}^{t} f_{k}\left(x_{i}\right)=f_{i}^{(t-1)}+f_{t}\left(x_{i}\right)$,

15 where $f_{t}\left(x_{i}\right)$ refers to the tree (learner) at step $\mathrm{t}, f_{i}^{(t)}$ and $f_{i}^{(t-1)}$ refer to the predicted values at steps $\mathrm{t}$ and $\mathrm{t}-1$, and $x_{i}$ is the input value.

$O b j^{(t)}=\sum_{k=1}^{n} l\left(\overline{y_{i}}, y_{i}\right)+\sum_{k=1}^{n} \Omega\left(f_{i}\right)$,

where $O b j^{(t)}$ is the regularized objective, $\overline{y_{i}}$ and $y_{i}$ refer to the prediction value and observed value, $l$ refers to the loss function, $n$ is the number of data set, and $\Omega$ refers to the regularization term, which equation is defined as follows:

$\Omega(f)=\gamma T+\frac{1}{2} \lambda\|\omega\|^{2}$,

where $\omega$ refers to the weight vector, $T$ denotes the total number of features, $\lambda$ is the regularization term, and $\gamma$ is the minimum loss.

\section{Supplementary Material}

\section{Section S2 Parameter adjustment of machine-learning models}

For the parameter adjustment in Table S2.1, for KNN, the kmax was 15; the distance was 1; the kernel was rectangular. For MLP, the size ranged between 5 and 10. For RF, the ntree was 1000; the mtry fluctuated from 9 to 11 . For SVM, the gamma was 0.01 ; the cost was 1 . For XGB, the max_depth was 3 -4 ; the eta was $0.05-0.1$; the colsample_bytree was $0.6-0.8$, the nrounds was 30 ; the subsample was $0.8-1$; the gamma was $0-0.4$; the min_child_weight was $0.6-0.8$. 
Table S2.1 Adjusted parameters for different machine-learning methods. "rectan" is short for rectangular, "opt" is short for optimal and "ep" is short for epanechnikov.

\begin{tabular}{|c|c|c|c|c|c|c|c|c|c|c|c|c|}
\hline Models & Parameters & alr1 & alr2 & clr1 & clr2 & $\operatorname{clr} 3$ & ilr1 & ilr2 & sand & silt & clay & class \\
\hline \multirow[t]{3}{*}{ KNN } & $\operatorname{kmax}$ & 13 & 13 & 14 & 14 & 15 & 15 & 14 & 14 & 15 & 15 & 15 \\
\hline & distance & 1 & 1 & 1 & 1 & 1 & 1 & 1 & 1 & 1 & 1 & 1 \\
\hline & kernel & rectan & rectan & rectan & rectan & rectan & rectan & opt & rectan & rectan & ep & rectan \\
\hline MLP & size & 5 & 5 & 5 & 5 & 5 & 5 & 5 & 10 & 10 & 10 & 5 \\
\hline \multirow[t]{2}{*}{$\mathrm{RF}$} & ntree & 1000 & 1000 & 1000 & 1000 & 1000 & 1000 & 1000 & 1000 & 1000 & 1000 & 1000 \\
\hline & mtry & 9 & 9 & 9 & 9 & 9 & 9 & 9 & 6 & 11 & 11 & 7 \\
\hline \multirow[t]{2}{*}{ SVM } & gamma & 0.1 & 0.1 & 0.1 & 0.1 & 0.1 & 0.1 & 0.1 & 0.1 & 0.1 & 0.1 & 0.01 \\
\hline & cost & 1 & 1 & 1 & 1 & 1 & 1 & 1 & 1 & 1 & 1 & 1 \\
\hline \multirow[t]{7}{*}{ XGB } & max_depth & 3 & 3 & 3 & 3 & 3 & 3 & 3 & 3 & 4 & 3 & 4 \\
\hline & eta & 0.1 & 0.1 & 0.1 & 0.1 & 0.1 & 0.1 & 0.1 & 0.05 & 0.05 & 0.05 & 0.1 \\
\hline & colsample_bytree & 0.6 & 0.6 & 1 & 0.8 & 0.6 & 0.6 & 1 & 0.6 & 0.6 & 0.6 & 0.8 \\
\hline & nrounds & 20 & 30 & 40 & 40 & 30 & 20 & 30 & 30 & 30 & 30 & 30 \\
\hline & subsample & 1 & 1 & 0.8 & 1 & 0.6 & 0.8 & 0.8 & 0.6 & 0.6 & 0.6 & 1 \\
\hline & gamma & 0.6 & 1 & 0.7 & 0.4 & 0.7 & 0 & 0.3 & 0.8 & 0.8 & 0.8 & 0.1 \\
\hline & min_child_weight & 0.6 & 0.8 & 0.6 & 1 & 0.6 & 1 & 1 & 0.8 & 0.8 & 0.8 & 0.6 \\
\hline
\end{tabular}




\section{Supplementary Material}

\section{Section S3 Uncertainty assessment of soil PSFs interpolation}

5 For the assessment of the uncertainties of models, Table S3.1 showed that ORI delivered lower SDs than those of log ratio methods among five machine-learning models for sand, silt and clay. Moreover, the ranges of $95 \%$ confidence interval (CI) of indicators were also computed, which indicated relatively low values compared with assessment indicators (Table S3.1). For KNN, MLP and RF, ORI method showed lower values of CI of RMSE, MAE and $\mathrm{R}^{2}$ than those of log ratio methods, and for SVM and

10 XGB, SVM_CLR and XGB_CLR revealed slight better performance compared with ORI of sand (CI_RMSE: $0.49 \%$; CI_MAE: $0.33 \%$ ) and silt (CI_MAE: $0.44 \%$ ), respectively. For the values of the ranges of $95 \% \mathrm{CI}$ of $\mathrm{AD}$ and STRESS, all models generated the same results (AD: 0.03, STRESS: 0.01) aside from RF_ILR (AD: 0.02), showing better performance. Thus, the estimators' variabilities had reasonable order of magnitudes for the values of the estimates and these indicators were representative 15 of the actual errors on independent test sets. 


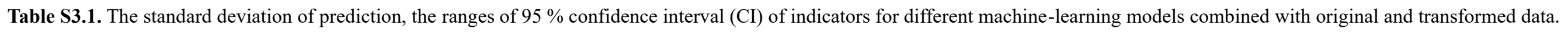

\begin{tabular}{|c|c|c|c|c|c|c|c|c|c|c|c|c|c|c|}
\hline & \multicolumn{3}{|c|}{ SD } & \multicolumn{3}{|c|}{ CI_RMSE (\%) } & \multicolumn{3}{|c|}{ CI_MAE (\%) } & \multicolumn{3}{|c|}{ CI_R ${ }^{2}(\%)$} & \multirow[t]{2}{*}{ CI_AD } & \multirow[t]{2}{*}{ CI_STRESS } \\
\hline & Sand & Silt & Clay & Sand & Silt & Clay & Sand & Silt & Clay & Sand & Silt & Clay & & \\
\hline KNN_ALR & 0.18 & 0.14 & 0.08 & 0.71 & 0.65 & 0.25 & 0.51 & 0.44 & 0.16 & 4.45 & 5.03 & 4.18 & 0.03 & 0.01 \\
\hline KNN_CLR & 0.18 & 0.14 & 0.08 & 0.71 & 0.64 & 0.26 & 0.47 & 0.41 & 0.16 & 4.57 & 4.95 & 4.23 & 0.03 & 0.01 \\
\hline KNN_ILR & 0.18 & 0.14 & 0.08 & 0.73 & 0.64 & 0.27 & 0.48 & 0.41 & 0.16 & 4.78 & 5.18 & 4.4 & 0.03 & 0.01 \\
\hline KNN_ORI & 0.15 & 0.11 & 0.07 & 0.55 & 0.51 & 0.28 & 0.38 & 0.37 & 0.19 & 3.41 & 3.48 & 4 & 0.03 & 0.01 \\
\hline MLP_ALR & 0.17 & 0.13 & 0.06 & 0.65 & 0.67 & 0.33 & 0.38 & 0.41 & 0.2 & 4.21 & 5.07 & 5.44 & 0.03 & 0.01 \\
\hline MLP_CLR & 0.16 & 0.13 & 0.06 & 0.64 & 0.65 & 0.32 & 0.38 & 0.41 & 0.19 & 4.07 & 4.96 & 5.12 & 0.03 & 0.01 \\
\hline MLP_ILR & 0.16 & 0.13 & 0.06 & 0.64 & 0.65 & 0.32 & 0.37 & 0.41 & 0.2 & 4.04 & 4.95 & 5.04 & 0.03 & 0.01 \\
\hline MLP_ORI & 0.15 & 0.11 & 0.06 & 0.65 & 0.58 & 0.23 & 0.37 & 0.4 & 0.17 & 3.72 & 4.02 & 2.72 & 0.03 & 0.01 \\
\hline RF_ALR & 0.18 & 0.15 & 0.08 & 0.62 & 0.54 & 0.25 & 0.42 & 0.38 & 0.17 & 4.03 & 3.91 & 4.03 & 0.03 & 0.01 \\
\hline RF_CLR & 0.18 & 0.15 & 0.07 & 0.66 & 0.64 & 0.27 & 0.42 & 0.42 & 0.18 & 4.25 & 4.45 & 4.12 & 0.03 & 0.01 \\
\hline RF_ILR & 0.18 & 0.15 & 0.08 & 0.69 & 0.66 & 0.27 & 0.44 & 0.42 & 0.18 & 4.34 & 4.75 & 4.31 & 0.02 & 0.01 \\
\hline RF_ORI & 0.15 & 0.12 & 0.07 & 0.53 & 0.54 & 0.25 & 0.4 & 0.41 & 0.16 & 2.95 & 3.47 & 3.06 & 0.03 & 0.01 \\
\hline SVM_ALR & 0.17 & 0.12 & 0.06 & 0.45 & 0.49 & 0.25 & 0.35 & 0.43 & 0.17 & 3.27 & 3.74 & 2.82 & 0.03 & 0.01 \\
\hline SVM_CLR & 0.16 & 0.12 & 0.06 & 0.49 & 0.5 & 0.27 & 0.33 & 0.35 & 0.18 & 3.05 & 3.35 & 3.47 & 0.03 & 0.01 \\
\hline SVM_ILR & 0.16 & 0.12 & 0.06 & 0.51 & 0.51 & 0.25 & 0.34 & 0.36 & 0.18 & 3.07 & 3.38 & 3.18 & 0.03 & 0.01 \\
\hline SVM_ORI & 0.15 & 0.11 & 0.06 & 0.51 & 0.49 & 0.25 & 0.34 & 0.35 & 0.17 & 2.92 & 3.14 & 2.95 & 0.03 & 0.01 \\
\hline XGB_ALR & 0.17 & 0.14 & 0.07 & 0.67 & 0.57 & 0.23 & 0.48 & 0.41 & 0.16 & 4.07 & 3.97 & 3.6 & 0.03 & 0.01 \\
\hline XGB_CLR & 0.19 & 0.15 & 0.07 & 0.73 & 0.65 & 0.25 & 0.44 & 0.44 & 0.16 & 4.9 & 5 & 3.82 & 0.03 & 0.01 \\
\hline XGB_ILR & 0.17 & 0.13 & 0.08 & 0.72 & 0.69 & 0.26 & 0.46 & 0.48 & 0.19 & 4.52 & 4.86 & 4.44 & 0.03 & 0.01 \\
\hline XGB_ORI & 0.16 & 0.12 & 0.06 & 0.6 & 0.61 & 0.24 & 0.41 & 0.46 & 0.16 & 3.4 & 4.03 & 2.9 & 0.03 & 0.01 \\
\hline
\end{tabular}


Supplementary Material

Section S4 Prediction maps of silt and clay fractions
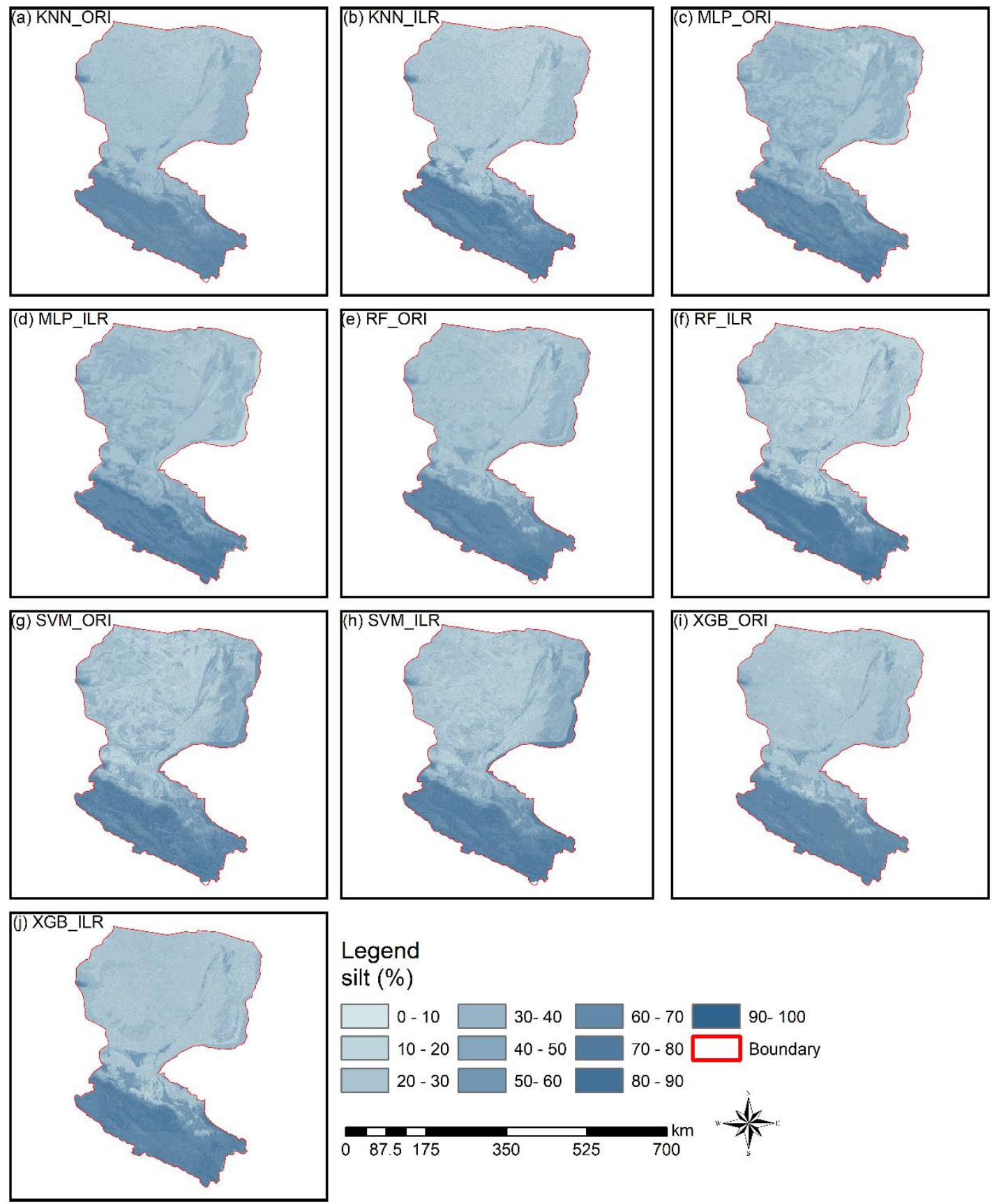

Figure S4.1. The prediction maps of silt fraction using five machine-learning models with ORI and ILR data. 

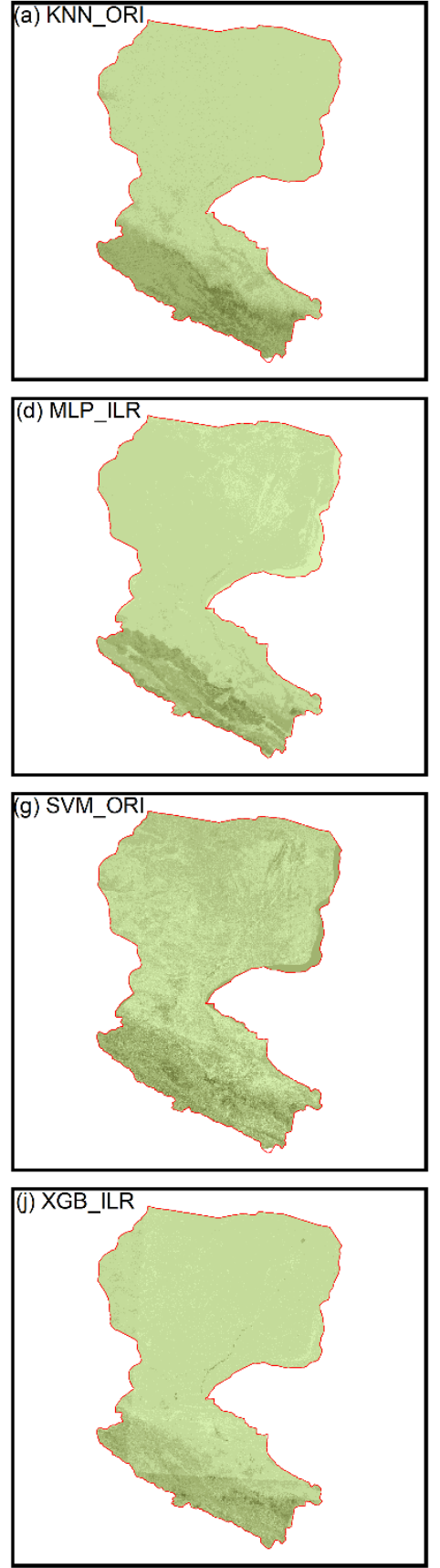

Legend

clay (\%)
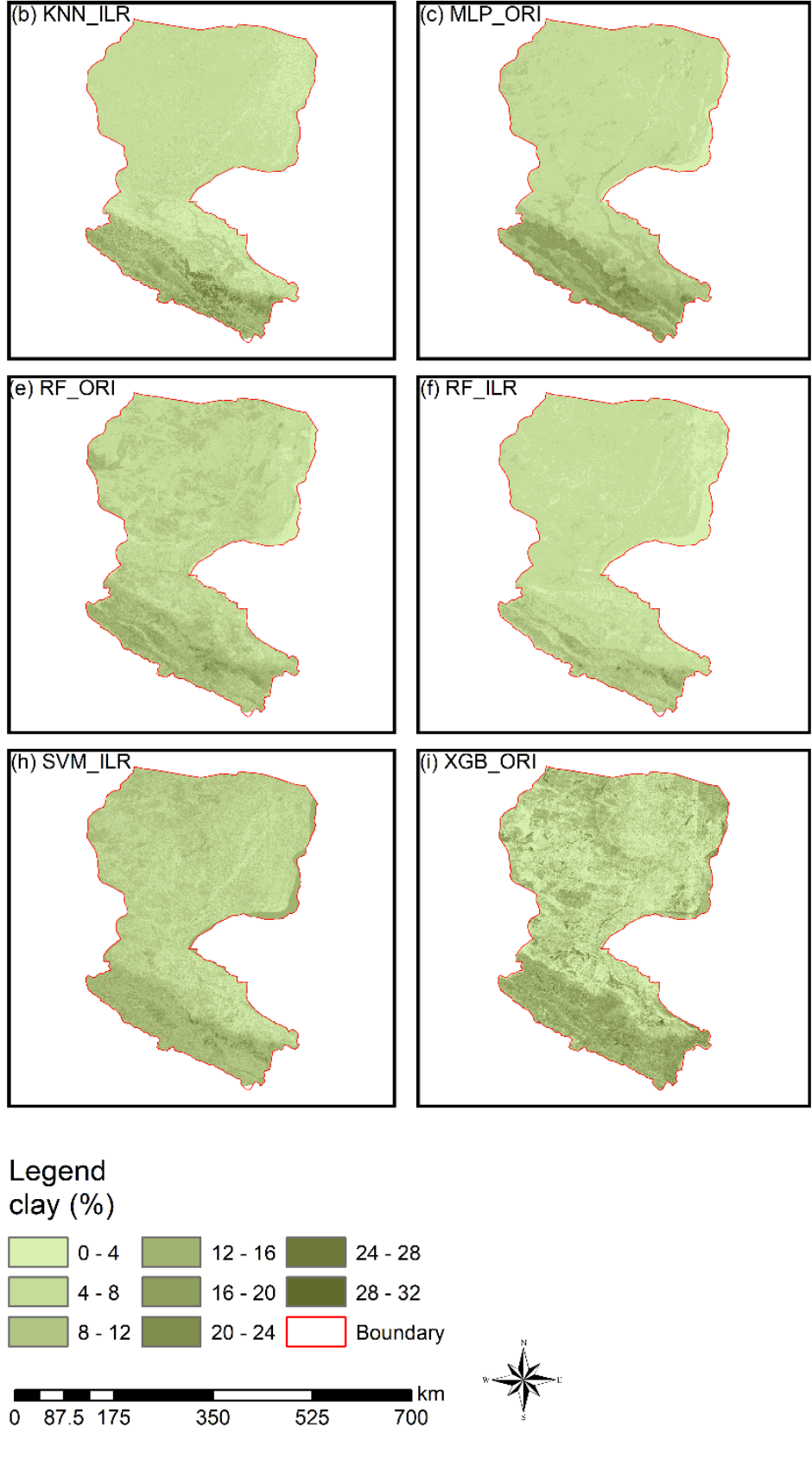

Figure S4.2. The prediction maps of clay fraction using five machine-learning models with ORI and ILR data. 
Supplementary Material

Section S5 Indirect classification maps using ALR and CLR transformation methods
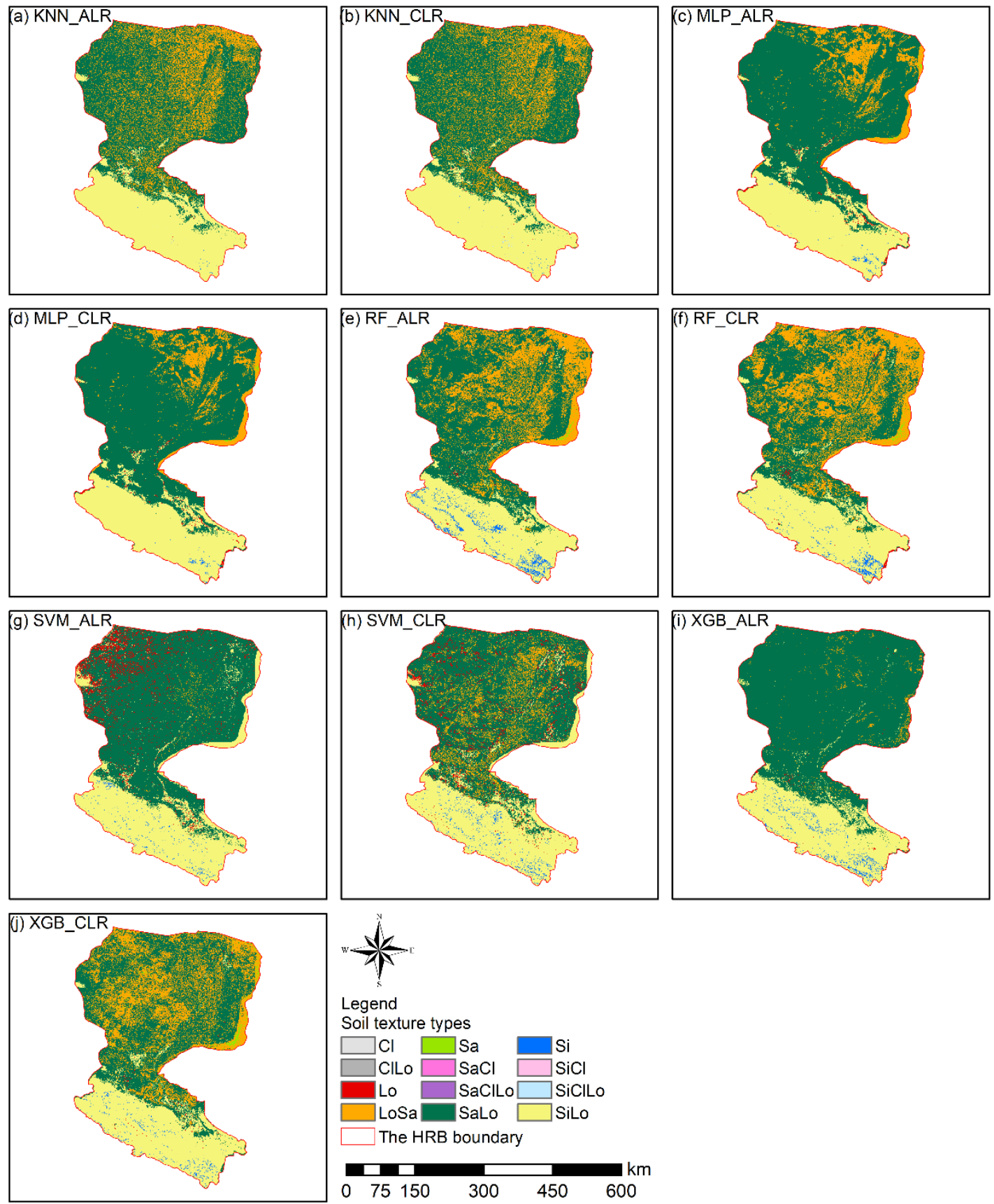

Figure S5.1. Soil texture classification prediction maps by soil PSFs interpolation (ALR and CLR log ratio transformation methods) of KNN, MLP, RF, SVM and XGB. 